\title{
Behaviour of moist and saturated sand during shock and release
}

\author{
J. I. Perry, C. H. Braithwaite, N. E. Taylor and A. P. Jardine \\ Cavendish Laboratory, JJ Thomson Avenue, Cambridge CB3 OHE, United Kingdom
}

Relatively little is known about the changes that occur in the shock compaction and release of granular matter with varying levels of moisture. Here, we report a series of plate impact experiments giving shock Hugoniot and release data for a well characterized sand at dry, $10 \%$ moist and saturated water contents. The results reveal that at low moisture content the shock impedance is slightly reduced, while the release remains predominantly inelastic. Close to saturation, much more substantial changes occur: the shock impedance stiffens substantially, the Hugoniot appears to split into two branches, and the release becomes almost completely elastic. We discuss mechanisms underpinning these changes in behavior.

Electronic mail: jip24@cantab.net

Granular systems are ubiquitous in the global environment and their behavior underpins a wide range of fundamental and technological phenomena. For example, fast dynamic compaction of sands and soils controls phenomena as diverse as interplanetary impact $^{1}$, seismic coupling ${ }^{2}$ and blast mitigation ${ }^{3}$. However, the inherent complexity of granular systems means that despite substantial research efforts over many decades, complete theoretical descriptions remain a substantial challenge ${ }^{4}$. Consequently, detailed empirical studies remain extremely important. To date, the shock compaction of dry sands has been studied in some detail ${ }^{5-8}$, and recently we established an approach that also provided information about the subsequent released state ${ }^{9,10}$. In this letter, we report a series of experiments probing the changes in shock compaction and release of a sand when varying amounts of moisture are included. We show that small amounts of moisture soften the response slightly, but that on saturation the mixture behaves considerably differently: the Hugoniot relationship splits into two branches, while the release becomes almost entirely elastic. Such changes are crucial to include in any high rate granular models, where it is important to represent realistic environmental conditions.

Dry granular materials are two-phase systems, where both bulk properties and the mesoscopic structure play roles in determining the dynamic response. The structure depends on factors including grain shape, size, porosity, surface roughness, arrangement and contact configuration ${ }^{11}$. It has not yet been possible to generalise shock behavior of dry sands across all these parameters, but several studies have used plate impact to establish the response of specific examples at up to about $2 \mathrm{GPa}^{5-8}$. For example, Chapman et al. ${ }^{6}$ provided the Hugoniot for a dry silica sand, while Brown et al. ${ }^{7}$ examined re-shocked states using VISAR. Similarly, the present authors obtained both the shock and release using a PDV based method ${ }^{9}$ to follow the rear face velocity of a sand sample, using a thin reflector that quickly rings up to stress equilibrium.

In contrast, there has been comparatively little research into shock compaction of wetted granular materials. The addition of moisture results in a threephase material with a substantially greater complexity; studies at lower strain rates have shown that a small amount of moisture can aid grain rearrangement through lubrication, but this is countered by a rate dependent increase in stiffness ${ }^{12,13}$. At full saturation the system returns to two phases, although the interstitial fluid then has substantial resistance to compression. Experiments on sandstone ${ }^{14}$ show a decrease in grain fracture in wetted samples. Dianov et al. ${ }^{15}$ performed early shock experiments that first showed saturated sand was significantly stiffer than dry, but they also saw a significant disparity between two grain fractions studied. Later, Chapman et al. ${ }^{16}$ obtained the Hugoniot of dry and moist sand, and in particular noted that close to saturation a small change in water content appeared to cause a surprisingly large increase in stiffness. However, the scatter in the observations limited the conclusions that could be drawn. More recently, Arlery et al. ${ }^{17}$ performed a small number of shock experiments using a small clay fraction to help stabilize and homogenize the system. The clay complicated the behaviour, making straightforward comparison more difficult. In all cases, the conclusions were limited by relatively small numbers of experiments, particularly given the statistical scatter in the results.

To determine the shock and release states of our sand/water samples, we used a method broadly similar to the approach we recently reported ${ }^{9}$. Onedimensional plate impact experiments were performed using the Cambridge plate impact facility ${ }^{18}$, which consists of a 2" bore single-stage light-gas gun, able to launch projectiles at velocities from 100 to $1000 \mathrm{~m} \mathrm{~s}^{-1}$, achieving planar impact with an angular precision of about $\pm 1 \mathrm{mrad}$. The experimental geometry is shown schematically in Fig. 1. PMMA target cells contain a 4 
$\mathrm{mm}$ bed of sand, which are impacted from the front using either copper (6 or $10 \mathrm{~mm}$ thick) or PMMA (10mm thick) flyer plates, to induce a state of onedimensional strain. A front make trigger consisting of two parallel copper strips of about $100 \mathrm{~nm}$ thickness was constructed by evaporating $99.99 \%$ pure copper onto the front surface of the cell in an Edwards 306 evaporator, minimizing the overall cell thickness. Impact by the copper flyer plate connects the two strips forming a short-circuit which is detected by a suitable measurement circuit. The small hole in the rear surface of the cell was closed with a $25 \mu \mathrm{m}$ thick copper foil. The foil quickly reaches stress equilibrium with the sand, and is monitored using a PDV laser velocimeter to provide the shock arrival time and free surface velocity, enabling the release path to be identified as described in $2013^{9}$.

The sand used is described as a "light brownorange, uniform, fine" builders' sand, and its particle size distribution is given in Fig. 2. The inset shows a typical VP-SEM image indicating the morphology. Rietveld quantitative analysis for the material (BraggBrentano geometry on a D8 Bruker diffractometer) gave compositions by weight of $(93.5 \pm 0.5) \% \alpha$-quartz and $(6.5 \pm 0.4) \%$ orthoclase. The material was sieved at $850 \mu \mathrm{m}$ to remove a small number of large inclusions and kiln dried for $24 \mathrm{~h}$ at $120^{\circ} \mathrm{C}$ to remove any residual moisture.

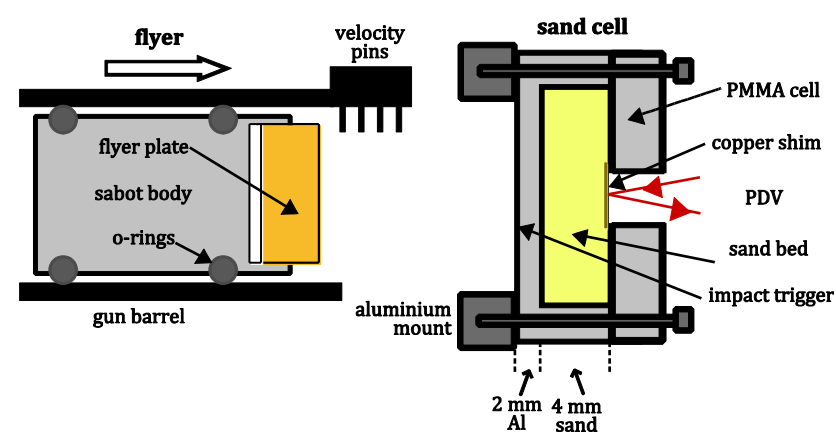

Figure 1: Schematic of the plate impact geometry and target cell (cross sectional view, not to scale). A copper or PMMA flyer plate impacts a PMMA cell containing a $4 \mathrm{~mm}$ sand bed. A front impact trigger and rear PDV measurement enable shock and release to be obtained, using the method described previously ${ }^{9}$.

Samples were prepared with three moisture contents (defined as the ratio of the component masses of each phase per unit volume of the composite material $\left.\rho_{\text {liquid }} / \rho_{\text {solid }}\right): 0 \%$ (dry), $(10 \pm 1) \%$ (moist), and $(23 \pm 2) \%$ (saturated). In the 10-20\% interval, we found that the mixture quickly separates under gravity into high and low moisture phases which cannot then be reliably used in the plate impact geometry. The dry sand cells were 'loosely poured' to a density of (1380-1450) $\mathrm{kg} \mathrm{m}^{-3}$. For the $10 \%$ moist samples, suitable quantities of sand and water were mixed in a 3-axis turbulent powder mixer.
From a large quantity of mixed material, the required quantity was placed into a cell, while a separate 'test sample' was weighed and dried to accurately check the moisture level in each experiment. The $10 \%$ moist sand was lightly manually compacted in the cells to (16001640) $\mathrm{kg} \mathrm{m}^{-3}$, (1450-1490) $\mathrm{kg} \mathrm{m}^{-3}$ of which is solid material. With the saturated material, this approach did not yield homogeneous samples. Instead, the cells were filled by adding small quantities of water followed by sand, in such a way that the sand remained oversaturated during the process. The mixture was stirred with a steel rod to ensure homogeneity, which had the additional benefit of lifting out residual air bubbles and resulted in a density of $(2000-2030) \mathrm{kg} \mathrm{m}^{-3}$. Other filling methods were examined, but resulted in visible air bubbles and a lower overall density. A test saturated sample was prepared and then baked dry, confirming the remaining air volume fraction was negligible $(<3 \%$, within experimental precision). The moisture content of the saturated cells $(23 \pm 2) \%$ could not be measured directly in each experiment, but was obtained from the total density and the assumption of zero remaining air. The cells were carefully sealed with epoxy resin to prevent water leakage under vacuum, and this technique was validated with several test cells.

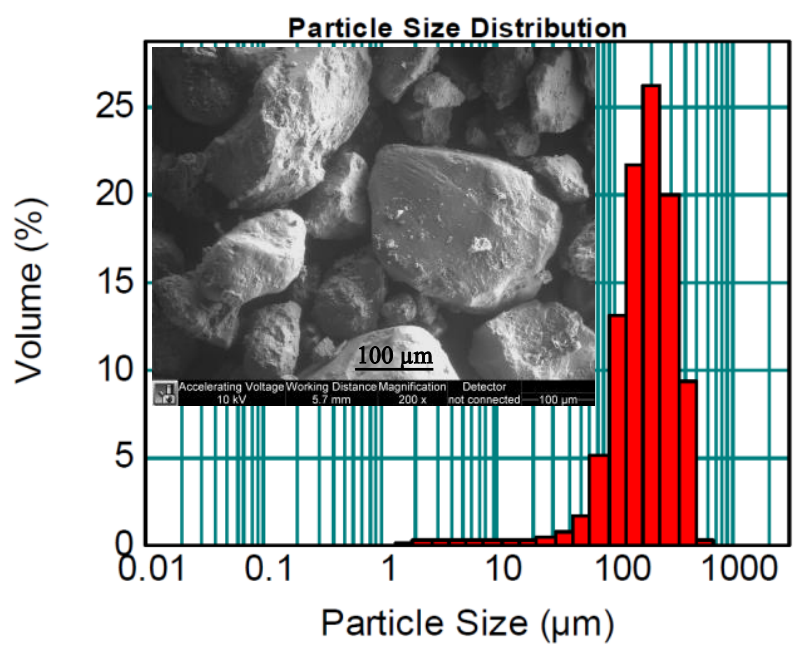

Figure 2: Particle size distribution for the sand used in this work, peaked at around $200 \mu \mathrm{m}$. Inset shows a VP-SEM image indicating the grain morphology.

From the experimental data, time-of-flight and impedance matching techniques were employed to give shock velocity, particle velocity and stress in both the PMMA and sand bed, using literature values for the Hugoniots of copper and PMMA ${ }^{19}$. Figure 3 compares the subsequent Hugoniot points obtained for the three moisture levels in shock-velocity $\left(\mathrm{U}_{\mathrm{s}}\right)$ - particlevelocity $\left(u_{p}\right)$ space, along with least squares linear fits.

Both dry and $10 \%$ moist sand show a linear $\mathrm{U}_{\mathrm{s}}-\mathrm{u}_{\mathrm{p}}$ relation, in common with many materials ${ }^{20}$. Although there is an increased scatter in the moist sand, which we attribute to the increased variability of the three phase mixture, it appears to have a small but consistently 
lower stiffness. All four data sets are confirmed as normally distributed about their linear fits (Shapiro$\mathrm{Wilk}^{21}$ ). Analysis of covariance $\left(\mathrm{ANCOVA}^{22}\right.$ ) gives the difference in gradient between dry and moist as negligible, and by using a common gradient to obtain an adjusted mean, the reduction in shock velocity due to an increase in moisture from 0 to $10 \%$ is $(191 \pm 43)$ $\mathrm{ms}^{-1}$. Therefore, there is a probability of $\mathrm{p}=0.0004$ that the two samples have the same Hugoniot.

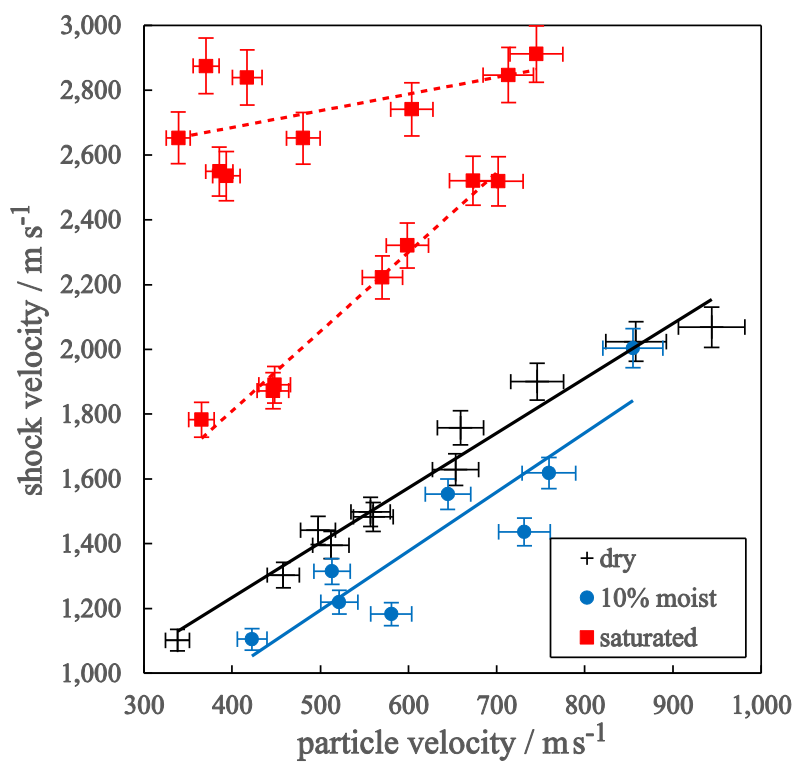

Figure 3: The Hugoniot points from each experiment, with best fit lines: $\mathrm{U}_{\mathrm{s}}=0.56+1.69 \mathrm{u}_{\mathrm{p}}\left(\mathrm{N}=11, \mathrm{R}^{2}=0.97\right)$ for dry, $\mathrm{U}_{\mathrm{s}}=0.36+1.72 \mathrm{u}_{\mathrm{p}}\left(\mathrm{N}=8, \mathrm{R}^{2}=0.82\right)$ for $10 \%$ moist, $\mathrm{U}_{\mathrm{s}}=$ $2.48+0.52 \mathrm{u}_{\mathrm{p}}\left(\mathrm{N}=9, \mathrm{R}^{2}=0.31\right)$ and $\mathrm{U}_{\mathrm{s}}=0.78+2.53 \mathrm{u}_{\mathrm{p}}(\mathrm{N}$ $=7, \mathrm{R}^{2}=0.98$ ) for saturated. $10 \%$ moist sand shows a small reduction in stiffness compared to dry sand. Saturated sand shows more complicated behavior, as described in the text.

This result is likely to have mesoscopic origins, as analysis of bulk properties alone would suggest that replacement of air with water, which is much stiffer and increases the overall density, should increase shock impedance ( since $\mathrm{Z}=\rho \mathrm{c}$ ). Two mechanisms are likely to contribute to the reduction in stiffness at low moisture contents. Firstly, small amounts of water will tend to sit at the points of grain-grain contact, reducing the peak stresses occurring at these interfaces ${ }^{1}$. The low sound speed in water and indirect paths through the granular system will then result in a softening/slowing of the overall shock. Secondly, when a material is loaded in plate impact, although the macroscopic strain is uniaxial, individual grains experience more complex loading. The overall material response is a combination of both the compression of the underlying material, as well as grain fracture and re-organization (compaction). Water will tend to lubricate the surface interactions between grains, facilitating compaction and can therefore reduce the overall stiffness.

For the saturated sand, corresponding to the upper set of points in Fig. 3, the shock response is significantly stiffer. Initially, the data appears to be quite widely scattered, but a closer inspection reveals the unexpected result that the Hugoniot points are split into two distinct lines. The effect is consistent, but only becomes apparent after performing a substantial number of experiments. Data for each of the two paths is scattered normally about the linear fit (Shapiro-Wilk), and, given our choice of splitting for the data (indicated in Fig 3), ANCOVA gives $p<0.0001$ for both sets of data having the same underlying Hugoniot. It is particularly surprising as the bulk properties of all the cells were very carefully controlled, for example to less than $2 \%$ variation in density. The fact that the bulk properties are so macroscopically similar suggests the effect has a microstructural origin. The suggestion is consistent with the decreasing divergence at very high stresses, when the material strength (and thus the microstructure) is less relevant, and only the equation of state remains important.

The filling mechanism we have used means that the samples are close to the boundary between being either (i) a fully saturated, but nevertheless interconnected network of sand grains surrounded by water; and (ii) a very dense particle (partial) suspension, with reduced inter-particle connectivity. These two phases appear to explain our observations. In the "interconnected-network" phase, all air has been replaced with water, but a quartz network remains that supports shock propagation, with a higher shock speed than water. The low compressibility of water compared to air suppresses stress-focusing at grain-grain interfaces and hence grain fracture and rearrangement. Compaction is therefore a much less significant process, and a flatter Hugoniot relationship is expected than for dry sand. Both these effects are consistent with the upper branch in our measurements.

In the "dense suspension" phase, compaction is also suppressed and similar behavior might be expected. However now the individual grains are separated by a thin layer of water, which has a lower shock impedance, and so the shockwave has to propagate through a series of grain-water-grain interfaces. These water bridges will reduce the shock speed slightly below that of the directly connected network. More importantly, however, at each interface the interstitial water has to 'ring-up' to stress equilibrium, requiring a number of wave reverberations. These result in a much longer effective path length and a lower shock speed, which is consistent with the lower branch of the data.

We now turn to the nature of the shock-release cycle for the three moisture contents, as shown in Fig. 4. Experimentally, we obtain the zero-pressure release point, which is connected linearly to the Hugoniot point, as justified in our previous paper". The dry release, shown in Fig. 4a, is predominantly inelastic as previously reported. At 10\% moisture, shown in Fig. $4 \mathrm{~b}$, we observe very similar inelastic behavior. In 
contrast, for saturated samples a very significantly different release behavior is seen; the release paths lie close to the Rayleigh loading line for the shock, resulting in a shock-release cycle which is approximately elastic. This indicates a more gradual stress attenuation with depth in a saturated granular sample, as energy dissipation is greatly reduced, in agreement with previous research on dry and saturated sandstone ${ }^{14}$.
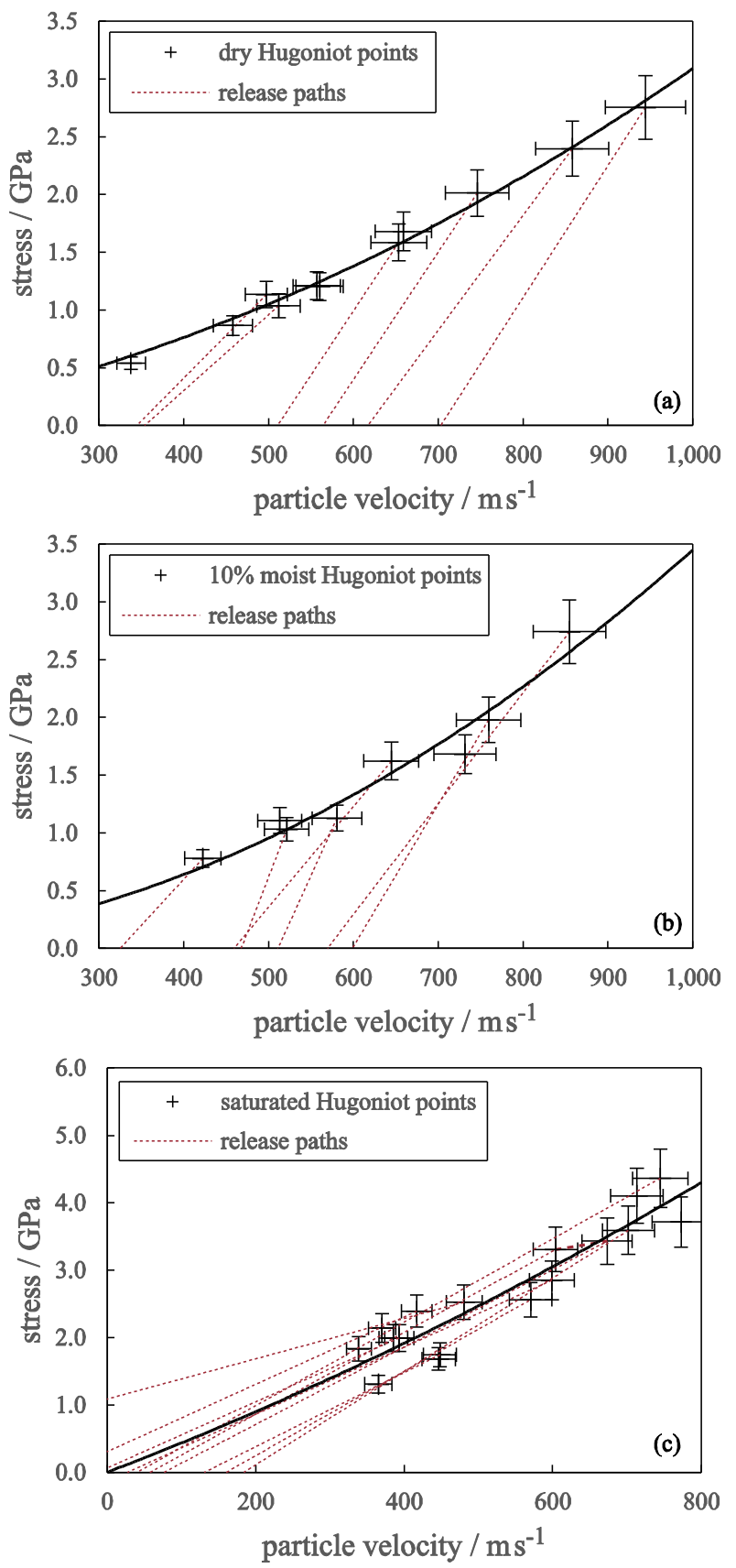

Figure 4: Shock data converted into stress-particle velocity space, showing the Hugoniots of (a) dry (reproduced from Appl. Phys. Lett. 103 (15), 154103 (2013). Copyright 2013 American Institute of Physics), (b) $10 \%$ moist and (c) saturated sands (with quadratic fit), including release paths obtained using the method described previously ${ }^{9}$. Both dry and $10 \%$ moist sand show a predominantly inelastic release, whereas for saturated sand the release is almost perfectly elastic.
The dominant inelastic processes for dry sand are grain fracture (enhanced by stress concentration at the inter-granular contact points), rearrangement and compaction. Elastic compression of individual grains is much less significant, and is the only process which contributes to re-assertion on release. At $10 \%$ moisture, the water bridges present between grains do not substantially modify such behavior. In contrast, the elastic release observed for saturated sand suggests that the primary mechanism occurring is elastic compression of both water and individual sand grains, and that the inelastic processes of grain fracture and rearrangement are almost insignificant. These conclusions, in combination with previous studies on sandstone ${ }^{14}$ appear to confirm that full water saturation suppresses grain fracture.

Taken together, our measurements provide substantial insight into the shock compaction of granular materials with increasing moisture content. The addition of small amounts of water has little effect on either shock or release. However, there is a major change around the point of saturation, including a substantially stiffer shock response, an elastic release, and a splitting into two Hugoniot branches. We suggest these branches are due to a sensitive dependence on the mesoscopic and microscopic structure of the material, with the different phases corresponding to either a saturated granular lattice or a very dense suspension. It will be interesting to explore whether these observations can be reproduced within microstructurally resolved numerical models.

We believe these insights are likely to be of wide importance. Changes in the shock-release cycle will significantly change the level of energy dissipation during shock compression of saturated sand, which will have significant consequences for many applications. Similarly, on large scales granular systems are often modelled at a continuum level; the observed behavior will be crucial to include in associated material models when realistic environmental conditions need to be accounted for. Finally, our present study relates to one well-characterised material. It will clearly be important to explore the generality of our observations, and particularly how the shock response is affected by particle size, morphology and adhesion, in order to provide a consistent description across the full spectrum of granular solids.

Acknowledgements: This work was supported through the Force Protection Engineering research programme led by QinetiQ Plc. on behalf of DSTL. 


\section{References}

1. E. Buhl, M. H. Poelchau, G. Dresen and T. Kenkmann, Meteoritics and Planetary Science 48, 71 (2013).

2. M. N. Toksöz, C. H. Cheng and A. Timur, Geophysics 41, 621 (1976).

3. T. Homae, K. Wakabayashi, T. Matsumura and Y. Nakayama, AIP Conference Proceedings 955, 1289 (2007).

4. R. Blumenfeld, S. F. Edwards and S. M. Walley, in The Oxford Handbook of Soft Condensed Matter, edited by E. M. Terentjev and D. A. Weitz (Oxford University Press, Oxford, 2015).

5. K. Tsembelis, W. G. Proud, B. A. M. Vaughan and J. E. Field, in Behaviour of Materials at High Strain Rates: Numerical Modelling, edited by F. G. Benitez (DYMAT, Saint-Louis, France, 2002), pp. 193 - 203.

6. D. J. Chapman, K. Tsembelis and W. G. Proud, AIP Conference Proceedings 845, 1445 (2005).

7. J. L. Brown, T. J. Vogler, D. E. Grady, W. D. Reinhart, L. C. Chhabildas and T. F. Thornhill, AIP Conference Proceedings 955, 1363 (2007).

8. D. J. Chapman, C. H. Braithwaite and W. G. Proud, AIP Conference Proceedings 955, 1367 (2007).

9. C. H. Braithwaite, J. I. Perry, N. E. Taylor and A. P. Jardine, Appl Phys Lett 103 (15), 154103 (2013).

10. J. I. Perry, C. H. Braithwaite, N. E. Taylor and A. P. Jardine, Journal of Physics: Conference Series 500, 112049 (2014).

11. X. Zheng and D. Wang, Acta Mechanica Solida Sinica 23, 579 (2010).

12. W. G. Proud, D. J. Chapman, D. M. Williamson, K. Tsembelis, J. Addiss, A. Bragov, A. Lomunov, I. G. Cullis, P. D. Church, P. Gould, D. Porter, J. R. Cogar and J. Borg, AIP Conference Proceedings 955, 1403 (2007).

13. M. Omidvar, M. Iskander and S. Bless, Int J Impact Eng 49, 192 (2012).

14. M. Hiltl, R. P. Swift, C. R. Hagelberg, T. C. Carney and W. J. Nellis, AIP Conference Proceedings 505, 1251 (1999).

15. M. D. Dianov, N. A. Zlatin, S. M. Mochalov, G. S. Pugachev and L. K. Rosomakho, Soviet Technical Physical Letters 2, 207 (1976).

16. D. J. Chapman, K. Tsembelis and W. G. Proud, in Annual Conference and Exposition on Experimental and Applied Mechanics (Society for Experimental Mechanics, Bethel CT, 2006).

17. M. Arlery, M. Gardou, J. M. Fleureau and C. Mariotti, Int J Impact Eng 37, 1 (2010).

18. N. K. Bourne, Z. Rosenberg, D. J. Johnson, J. E. Field, A. E. Timbs and R. P. Flaxman, Measurement Science and Technology 6, 1462 (1995).

19. S. P. Marsh, LASL Shock Hugoniot data. (University of California Press, Los Angeles, CA, 1980).
20. J. Asay and M. Shahinpoor, High-pressure shock compression of solids. (Springer-Verlag, New York, 1993).

21. S. S. Shapiro and M. B. Wilk, Biometrika 52 (3-4), 591-611 (1965).

22. D. C. Montgomery, Design and Analysis of Experiments, 8th ed. (John Wiley \& Sons, 2012). 